\title{
Gauge invariance of transverse momentum dependent distributions at small $x$
}

\author{
Renaud Boussarie* \\ Los Alamos National Laboratory, Theoretical Division, Group T-2, Los Alamos, New Mexico 87545, USA \\ and Physics Department, Brookhaven National Laboratory, Upton, New York 11973, USA \\ Yacine Mehtar-Tani ${ }^{\dagger}$ \\ RIKEN BNL Research Center and Physics Department, Brookhaven National Laboratory, \\ Upton, New York 11973, USA
}

(Received 16 November 2020; accepted 8 February 2021; published 13 May 2021)

\begin{abstract}
The interplay between the small- $x$ limit of QCD amplitudes and QCD factorization at moderate $x$ has been studied extensively in recent years. It was finally shown that semiclassical formulations of small- $x$ physics can have the form of an infinite twist framework involving transverse momentum dependent distributions in the eikonal limit. In this work, we demonstrate that small- $x$ distributions can be formulated in terms of transverse gauge links. This allows, in particular, for direct and efficient decompositions of observables into subamplitudes involving gauge-invariant suboperators which span parton distributions.
\end{abstract}

DOI: 10.1103/PhysRevD.103.094012

\section{INTRODUCTION}

The two main regimes for a process with a hard scale $Q^{2}$ and center-of-mass energy $s$ are the so-called Bjorken limit $Q^{2} \sim s \rightarrow \infty$ that relates to moderate values of $x \sim Q^{2} / s$, where QCD factorization applies, and the so-called Regge limit, for which $Q^{2} \ll s$ or $x \ll 1$. The main obstacle when studying the connections between QCD factorization and QCD at small $x$ is the discrepancy in the involved nonperturbative elements: QCD factorization involves parton distributions, whereas the most general formulations of small- $x$ physics involve the action of Wilson-line operators on hadronic states [1-6]. In Ref. [7], the authors showed how to extract the small- $x$ limit of a transverse momentum dependent (TMD) distribution from a Wilson-line operator. Their argument relies on two statements: the fact that the longitudinal momentum fractions of partons in the distributions are small in the eikonal limit and the fact that transverse gluon fields are subeikonal in the most commonly used gauges (see Sec. II).

As an example, let us consider the WeizsäckerWilliams (WW)-type gluon TMD operator for a gluon with longitudinal momentum fraction $x$ and transverse

\footnotetext{
*renaud@lanl.gov

†mehtartani@bnl.gov
}

Published by the American Physical Society under the terms of the Creative Commons Attribution 4.0 International license. Further distribution of this work must maintain attribution to the author(s) and the published article's title, journal citation, and DOI. Funded by SCOAP ${ }^{3}$. momentum $\boldsymbol{k}$ [8] inside a hadron with a large momentum $P$ mostly in the - light cone direction, which is defined as [9]

$$
\mathcal{O}^{i j}(x, \boldsymbol{k}) \equiv \int \frac{\mathrm{d} r^{+} \mathrm{d}^{2} \boldsymbol{r}}{(2 \pi)^{3}} \mathrm{e}^{i x P^{-} r^{+}-i(\boldsymbol{k} \cdot \boldsymbol{r})} F^{i-}(r) \mathcal{U}_{[r, 0]}^{[+]} F^{j-}(0) \mathcal{U}_{[0, r]}^{[+]},
$$

where [10] $F^{i-} \equiv \partial^{i} A^{-}-\partial^{-} A^{i}-i g\left[A^{i}, A^{-}\right]$[cf. Eq. (8)] is the field strength tensor, $\mathcal{U}_{[r, 0]}^{[ \pm]}$are staple-shaped gauge links

$$
\mathcal{U}_{[x, y]}^{[ \pm]}=\left[x^{+}, \infty^{+}\right]_{x}[\boldsymbol{x}, \boldsymbol{y}]_{ \pm \infty^{+}}\left[ \pm \infty^{+}, y^{+}\right]_{\boldsymbol{y}},
$$

and $\left[x^{+}, \infty^{+}\right]_{x}$ denotes a straight Wilson line in the fundamental representation of $\mathrm{SU}(3)$ along the + direction at fixed transverse coordinate $\boldsymbol{x}$ and similarly for $\left[ \pm \infty^{+}, y^{+}\right]_{y}$. In particular, we have

$\left[x^{+}, y^{+}\right]_{x}=U_{x}\left(x^{+}, y^{+}\right) \equiv \mathcal{P} \exp \left[i g \int_{y^{+}}^{x^{+}} \mathrm{d} z^{+} A^{-}\left(z^{+}, \boldsymbol{x}\right)\right]$,

where $A^{-}\left(x^{+}, \boldsymbol{x}\right)=n \cdot A$ is the - component of the target gauge field evaluated on the light cone branch $x^{-}=0$, with $n=(1,0,0,1)$. Here and throughout, it is understood that $A \equiv A^{a} t^{a}$, where $t^{a}$ are the QCD color matrices in the fundamental representation.

The transverse gauge link, on the other hand, is evaluated at constant light cone time $x^{+}$: 


$$
[\boldsymbol{x}, \boldsymbol{y}]_{x^{+}} \equiv \mathcal{P} \exp \left[-i g \int_{\boldsymbol{y}}^{x} \mathrm{~d} \boldsymbol{z}(\lambda) \cdot \boldsymbol{A}\left(x^{+}, \boldsymbol{z}(\lambda)\right)\right]
$$

where $z(\lambda) \equiv\left(z^{1}, z^{2}\right)$ defines a trajectory in the transverse plane, that starts at $\boldsymbol{y}$ and ends at $\boldsymbol{x}$, and parametrized by the real number $0<\lambda<1$.

Under the two hypotheses described above, one can neglect the phase $i x P^{-} r^{+}$in the Fourier transform in Eq. (1), as well as the transverse part $[\boldsymbol{x}, \boldsymbol{y}]_{\infty^{+}}$of the stapleshaped gauge link. This observation allowed the authors of Ref. [9] to find a match between the WW-type TMD operator and infinite Wilson-line operators $U_{x} \equiv$ $U_{x}(\infty,-\infty)$. Indeed,

$$
\mathcal{O}^{i j}(x \approx 0, \boldsymbol{k}) \propto \int \mathrm{d}^{2} \boldsymbol{r} \mathrm{e}^{-i(\boldsymbol{k} \cdot \boldsymbol{r})} U_{\boldsymbol{r}}\left(\partial^{i} U^{\dagger}\right)_{\boldsymbol{r}} U_{\mathbf{0}}\left(\partial^{j} U^{\dagger}\right)_{\mathbf{0}} .
$$

Such an operator appears naturally when one takes the first term in Taylor expansions of observables at small $x$. The remarkable equivalence in Eq. (5) generated a lot of interest for the physics of TMD distributions in the small- $x$ community, which gathered tremendous insight on these distributions from small- $x$ models [11-18]. Attempts have also been made in order to unify small- $x$ and moderate- $x$ evolution equations for TMDs [19,20]. However, no equivalence was formed beyond the leading power in $\boldsymbol{k} / Q$ until very recently [21,22].

In Refs. [21,22], it was shown that a class of observables at small $x$ could be entirely rewritten as the eikonal limit of an infinite twist TMD framework. This new formulation of small- $x$ physics was based on a power expansion, then the rearrangement of the expanded form by classifying terms depending on the genuine twist of the nonperturbative operator involved, and then the resummation of power corrections to the accompanying Wilson coefficients. Although the final expressions were fairly simple, it would be cumbersome to generalize to other classes of observables. It also relied on the assumption that neglecting the transverse gauge links from the distribution in a gauge where transverse gluons are subeikonal would not spoil QCD gauge invariance of the distribution.

In this article, we propose a more direct derivation of the equivalence found in Refs. [21,22] and uncover its underlying geometric structure which preserves the explicit gauge invariance of the operators. For this purpose, we demonstrate that pairs of Wilson-line operators have a powerful formulation in terms of transverse gauge links built from rotated gluon fields. In the new approach to TMDs at small $x$ in terms of transverse gauge link operators, it will be straightforward to generalize to other observables.

The article is organized as follows: In Sec. II, we give a brief introduction of the semiclassical small- $x$ basics and discuss gauge invariance for this framework. In Sec. III, we show how pairs of Wilson lines can be interpreted as transverse gauge links thanks to parallel transports on the transverse plane and gauge invariance and how this result has the form of a non-Abelian Stokes equation. As an application of the results from Sec. III, we show how to extract TMD suboperators from the dipole in Sec. IV, and in Sec. V we extend this method to more generic two-Wilsonline operators. Finally, we extend the method to a three-line operator in Sec. VI.

\section{BACKGROUND FIELD AT HIGH ENERGY}

Consider a hadronic target moving in the negative $z$ direction, close to the light cone, i.e., $x^{-} \sim 0$. It can be described by a classical current $[3,23]$

$$
J^{-}(x) \approx J^{-}\left(x^{+}, \boldsymbol{x}\right) \text { and } J^{+} \approx J^{i} \approx 0,
$$

that generates a gauge field which depends only on light cone time $x^{+}$and the transverse coordinate $\boldsymbol{x}$. In such a framework, it turns out that both covariant $\partial \cdot A=0$ and light cone $A^{+}=0$ gauge share a common solution. Indeed, it immediately follows from $A^{+}=A^{i}=0$ and the independence on $x^{-}$that $\partial \cdot A=\partial^{+} A^{-}=0$. The equation of motion for the field reads

$$
\left[D_{\mu}, F^{\mu-}\right]=-\partial^{i} F^{i-}=-\partial^{2} A^{-}=J^{-},
$$

where

$F^{\mu \nu} \equiv \partial^{\mu} A^{\nu}-\partial^{\nu} A^{\mu}-i g\left[A^{\mu}, A^{\nu}\right] \quad$ and $\quad D^{\mu} \equiv \partial^{\mu}-i g A^{\mu}$

denote the field strength tensor and the covariant derivative, respectively. With the above choice of gauges, the current is covariantly conserved, since $D^{+} J^{-}=$ $\partial^{+} J^{-}=0$. Furthermore, note that, although $A^{-}$obeys a Poisson equation, it is an exact solution of the Yang-Mills equations.

In the following, we will make use of the existence of a residual gauge freedom in $A^{+}=0$ light cone gauge: One can choose any gauge transformations with the form

$$
\begin{aligned}
A^{-}\left(x^{+}, \boldsymbol{x}\right) \rightarrow & \Omega_{x}\left(x^{+}\right) A^{-}\left(x^{+}, \boldsymbol{x}\right) \Omega_{x}^{-1}\left(x^{+}\right) \\
& -\frac{1}{i g} \Omega_{x}\left(x^{+}\right) \partial^{-} \Omega_{x}^{-1}\left(x^{+}\right), \\
A^{i}\left(x^{+}, \boldsymbol{x}\right) \rightarrow & -\frac{1}{i g} \Omega_{x}\left(x^{+}\right) \partial^{i} \Omega_{x}^{-1}\left(x^{+}\right),
\end{aligned}
$$

where $\Omega_{x}\left(x^{+}\right)$is an element of the gauge group SU(3) that preserves the condition $A^{+}=0$. Because $A^{+}$is suppressed on the $x^{-}=0$ branch, the above decomposition is expected to span a larger subset of $\mathrm{SU}(3)$ beyond light cone gauge. 
Under such a gauge transformation, the Wilson line

$$
U_{\boldsymbol{x}}\left(\xi_{2}, \xi_{1}\right)=\mathcal{P} \exp \left[i g \int_{\xi_{1}}^{\xi_{2}} \mathrm{~d} x^{+} A^{-}\left(x^{+}, \boldsymbol{x}\right)\right]
$$

transforms as

$$
U_{x}\left(\xi_{2}, \xi_{1}\right) \rightarrow \Omega_{x}\left(\xi_{2}\right) U_{x}\left(\xi_{2}, \xi_{1}\right) \Omega_{x}^{-1}\left(\xi_{1}\right) .
$$

Note that there is an infinite number of gauge transformations, spanning the entire gauge group $\mathrm{SU}(3)$, that satisfy (9). This freedom in fixing the boundary conditions translates into the different regularization schemes for the spurious $1 / k^{+}$singularity encountered in light cone gauge; see, for example, Ref. [24].

\section{TRANSVERSE QCD STRINGS AND THE DIPOLE OPERATOR}

In the standard semiclassical formulations of small- $x$ physics, scattering amplitudes are most commonly computed in $A^{+}=0$ light cone gauge by fixing the residual gauge freedom such that the classical transverse field vanishes. Hence, only the - component of the background field is taken into account through lightlike Wilson-line operators along the $x^{+}$direction.

The connection to TMD physics requires the formulation of the problem in terms of field strength tensors, typically introduced by performing a gradient expansion in transverse coordinate space that yields $\partial^{i} A^{-} \sim F^{i-}$. In effect, this corresponds to the parallel transport of Wilson-line operators on the transverse plane as will be shown shortly.

\section{A. Parallel transport on the transverse plane}

Let us first show how gauge rotations can be rewritten as transverse gauge links. For any pair of transverse positions $\left(\boldsymbol{x}_{1}, \boldsymbol{x}_{2}\right)$ and defining $\boldsymbol{r}=\boldsymbol{x}_{1}-\boldsymbol{x}_{2}$, we can readily write [25]

$$
\begin{aligned}
\Omega_{x_{1}}(t) & =\Omega_{x_{2}}(t)+\int_{0}^{1} \mathrm{~d} s \frac{\mathrm{d}}{\mathrm{d} s} \Omega_{x_{2}+s r}(t) \\
& =\Omega_{\boldsymbol{x}_{2}}(t)-r^{i} \int_{0}^{1} \mathrm{~d} s\left(\partial^{i} \Omega\right)_{\boldsymbol{x}_{2}+s \boldsymbol{r}}(t) .
\end{aligned}
$$

Now, note that

$$
\partial^{i} \Omega_{x}(t)=i g A^{i}(t, x) \Omega_{x}(t),
$$

where $A^{i}$ (with $i=1,2$ ) is the pure gauge field obtained from the rotation $\Omega$ [see Eq. (9)]. Combining the two remarks above and then multiplying Eq. (12) by $\Omega_{x_{2}}^{-1}(t)$ on the right yields

$$
\Omega_{x_{1}}(t) \Omega_{x_{2}}^{-1}(t)=1-i g r^{i} \int_{0}^{1} \mathrm{~d} s A^{i}\left(t, \boldsymbol{x}_{2}+s \boldsymbol{r}\right) \Omega_{x_{2}+s \boldsymbol{r}}(t) \Omega_{x_{2}}^{-1}(t) .
$$

The final step is now to notice that Eq. (14) is the equation which defines a Wilson line along the straight line trajectory parametrized by the real number $s$ with values between 0 and 1 and such that $z(0)=x_{2}$ and $z(1)=x_{1}$, that is,

$$
z(s)=\boldsymbol{x}_{2}+s \boldsymbol{r} .
$$

This Wilson line, that we denote as $\left[\boldsymbol{x}_{1}, \boldsymbol{x}_{2}\right]_{t}$, solves the following equation:

$$
\left[x_{1}, x_{2}\right]_{t}=1-i g \int_{x_{2}}^{x_{1}} \mathrm{~d} z \cdot \boldsymbol{A}(t, z)\left[z, x_{2}\right]_{t} .
$$

Although the transverse gauge link was constructed for a straight line trajectory, it can be easily shown that it is independent of the trajectory connecting the end points so long as the transverse field is a pure gauge.

By comparing Eqs. (14) and (15), we can make the following identification:

$$
\Omega_{x_{1}}(t) \Omega_{x_{2}}^{-1}(t)=\left[x_{1}, x_{2}\right]_{t} .
$$

An equivalent relation can readily be obtained:

$$
\left[x_{1}, x_{2}\right]_{t}=1-i g \int_{x_{2}}^{x_{1}}\left[x_{1}, z\right]_{t} A(t, z) \cdot \mathrm{d} z .
$$

\section{B. Dipole operator and the non-Abelian Stokes theorem}

With the setup from Sec. II, where transverse gluon fields are neglected, let us consider a generic dipole operator in the fundamental representation:

$$
\left(\mathcal{O}_{\xi}\left(\boldsymbol{x}_{1}, \boldsymbol{x}_{2}\right)\right)_{i j} \equiv\left(U_{\boldsymbol{x}_{1}}(\infty, \xi) U_{\boldsymbol{x}_{2}}(\xi, \infty)\right)_{i j},
$$

where $i, j$ are color indices. In most cases, at small $x$ it is assumed that the classical field $A^{-}$has a compact support which is very peaked around $x^{+}=0$ in Eq. (10). As a result, so long as $\xi<0$, one can replace $\xi$ by $-\infty$ in our expressions, and one can deal with infinite Wilson lines $U_{\boldsymbol{x}}$. In Eq. (18), this choice would amount to the replacement [26] $U_{x_{1}}(\infty, \xi) U_{x_{2}}(\xi, \infty) \rightarrow U_{x_{1}} U_{x_{2}}^{\dagger}$. Here, we will keep a generic $\xi$. Under the gauge rotation of parameter $\Omega$, the dipole operator becomes

$$
\begin{aligned}
\mathcal{O}_{\xi}\left(x_{1}, x_{2}\right) \rightarrow & \Omega_{x_{1}}^{-1}(\infty) U_{x_{1}}(\infty, \xi) \Omega_{x_{1}}(\xi) \Omega_{x_{2}}^{-1}(\xi) \\
& \times U_{x_{2}}(\xi, \infty) \Omega_{x_{2}}(\infty),
\end{aligned}
$$

where the lines are now built from gauge-rotated gluon fields from Eq. (9). Using Eq. (16) finally allows us to write the dipole operator with transverse gauge links:

$$
\mathcal{O}_{\xi}\left(x_{1}, x_{2}\right)=\Omega_{x_{1}}^{-1}(\infty) U_{x_{1}}(\infty, \xi)\left[x_{1}, x_{2}\right]_{\xi} U_{x_{2}}(\xi, \infty) \Omega_{x_{2}}(\infty) .
$$



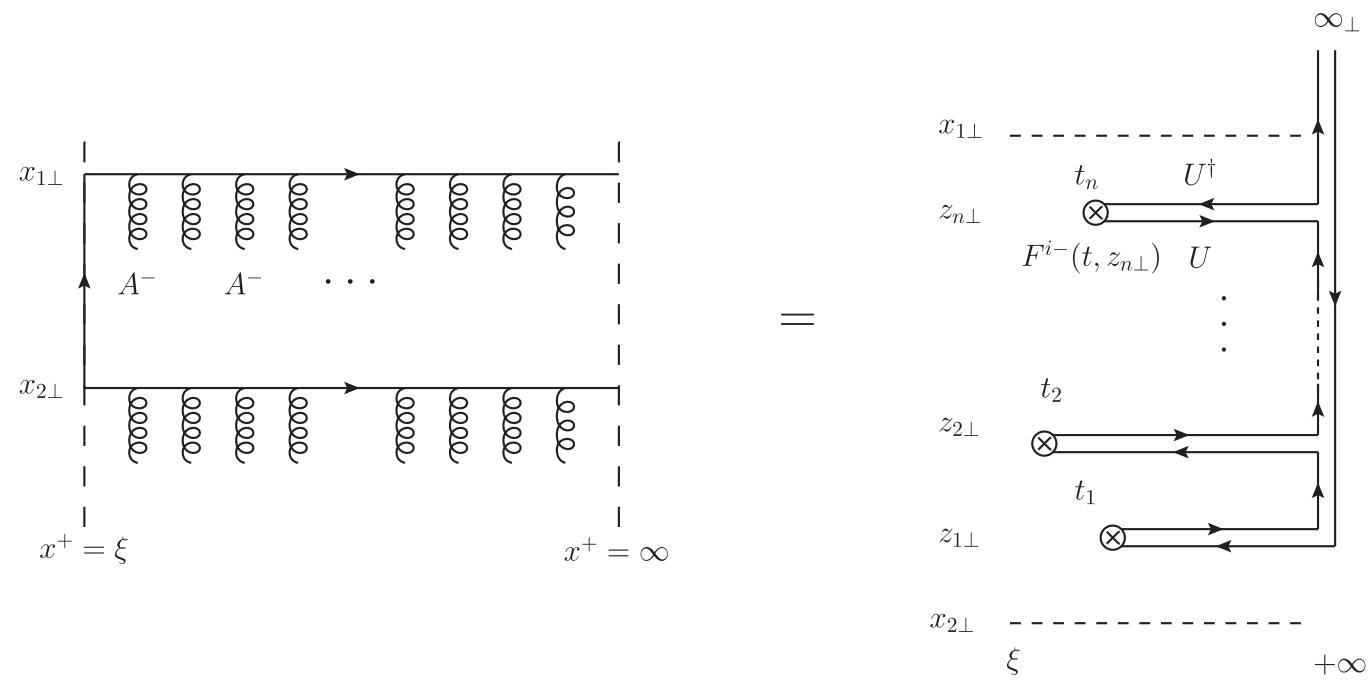

FIG. 1. Non-Abelian Stokes theorem.

A diagrammatic depiction of $\mathcal{O}_{\xi}\left(\boldsymbol{x}_{1}, \boldsymbol{x}_{2}\right)$ is given in Fig. 1, left panel. In Eq. (20), the transverse gauge link is built from the pure gauge transverse gluons $A^{i}\left(x^{+}, \boldsymbol{x}\right)=$ $-\frac{1}{i g} \Omega_{x}\left(x^{+}\right) \partial^{i} \Omega_{x}^{-1}\left(x^{+}\right)$.

It is possible to absorb the longitudinal Wilson lines into the transverse link with the following change of variables:

$$
\begin{aligned}
A^{i}(\xi, z) \rightarrow & \hat{A}^{i}(\xi, z) \equiv U_{z}(\xi, \infty) A^{i}(\xi, z) U_{z}(\xi, \infty) \\
& +\frac{1}{i g}\left(\partial^{i} U_{z}\right)(\infty, \xi) U_{z}(\xi, \infty) .
\end{aligned}
$$

Note that the hatted field is nonlocal in $x^{+}$and transforms similarly to the field strength tensor under a gauge rotation, i.e.,

$$
\hat{\boldsymbol{A}}(\xi, z) \rightarrow \Omega_{z}(\infty) \hat{\boldsymbol{A}}(\xi, z) \Omega_{z}^{-1}(\infty) .
$$

We can actually trade the dependence on the gauge field with that of the field strength tensor with simple algebra:

$$
\begin{aligned}
\hat{A}^{i}(\xi, z) & =\int_{\xi}^{\infty} \mathrm{d} t \frac{\mathrm{d}}{\mathrm{d} t} \hat{A}^{i}(t, z)+A^{i}(\infty, z) \\
& =\int_{\xi}^{\infty} \mathrm{d} t U_{z}(\infty, t) F^{i-}(t, z) U_{z}(t, \infty)+A^{i}(\infty, z),
\end{aligned}
$$

where we have used Eq. (21) and the fact that $(\partial / \partial t) \times$ $U_{z}(\infty, t)=-i g U_{z}(\infty, t) A^{-}(t, z) \quad$ and $\quad(\partial / \partial t) U(t, \infty)=$ $i g A^{-}(t, z) U_{z}(t, \infty)$. Furthermore, by making use of the Fierz identity

$$
U \mathbf{t}^{a} U^{\dagger}=\mathbf{t}^{b} W^{b a},
$$

one can also express Eq. (25) in terms of a single Wilson line $W^{b a}$ in the adjoint representation:

$\hat{A}^{i}(\xi, z)=\int_{\xi}^{\infty} \mathrm{d} t \mathbf{t}^{b} W_{z}^{b a}(\infty, t) F^{a, i-}(t, z)+A^{i}(\infty, z)$.

Without loss of generality, since transverse gluon fields are pure gauges, it is possible to chose the gauge rotation such that $A^{i}(\infty, z)=0$, simply by choosing $\Omega_{x}(\infty)=1$ on the whole transverse plane. We will make this choice from now on. For the sake of clarity, we will distinguish the gauge links which depend on the rotated field from Eqs. (21) and (25) from the regular gauge links by hatting their coordinates. We have now established that

$$
\mathcal{O}_{\xi}\left(\boldsymbol{x}_{1}, \boldsymbol{x}_{2}\right)=\left[\hat{\boldsymbol{x}}_{1}, \hat{\boldsymbol{x}}_{2}\right]_{\xi},
$$

which allows one to understand the dipole operator as a transverse string built from the gluons in Eq. (25).

We can rewrite this result in the form of the non-Abelian Stokes theorem ([27]; see also [28]): The integral of $A^{\mu}$ over the square contour $\mathcal{C}$ on the left-hand side in Fig. 1 is equal to the integral of the so-called twisted strength tensor $U F^{\mu \nu} U^{\dagger}$ inside the surface $\mathcal{S}$ defined by this contour:

$$
\mathcal{S}=\left\{(t, z) ; t \in[\xi, \infty], z \in\left[\boldsymbol{x}_{1}, \boldsymbol{x}_{2}\right]\right\} .
$$

Indeed, the more explicit Eq. (26), with nonvanishing transverse fields at infinite light cone time, is given by

$$
\begin{aligned}
& \left(\mathcal{P} \mathrm{e}^{i g \int_{\xi}^{\infty} \mathrm{d} t A^{-}\left(t, \boldsymbol{x}_{1}\right)} \mathcal{P}^{-i g \int_{x_{2}}^{x_{1}} \mathrm{~d} z \cdot \boldsymbol{A}(\xi, z)} \mathcal{P} \mathrm{e}^{i g \int_{\xi}^{\infty} \mathrm{d} t A^{-}\left(t, \boldsymbol{x}_{2}\right)} \mathcal{P} \mathrm{e}^{-i g \int_{x_{1}}^{x_{2}} \mathrm{~d} \cdot \boldsymbol{A}(\infty, z)}\right)^{i j} \\
& \quad=\left(\mathcal{P} \exp \left[-i g \int_{\boldsymbol{x}_{2}}^{\boldsymbol{x}_{1}} \mathrm{~d} \boldsymbol{z}^{i} \int_{\xi}^{\infty} \mathrm{d} t\left[\boldsymbol{x}_{1}, \boldsymbol{z}\right]_{\infty}[\boldsymbol{\infty}, t]_{z} F^{i-}(t, \boldsymbol{z})[t, \infty]_{z}\left[\boldsymbol{z}, \boldsymbol{x}_{1}\right]_{\infty}\right]\right)^{i j},
\end{aligned}
$$


which is a form of the Stokes equation

$\mathcal{P} \exp \left[\oint_{\mathcal{C}} \mathrm{d} x_{\mu} A^{\mu}(x)\right]=\mathcal{P} \exp \left[\int_{\mathcal{S}} \mathrm{d} \sigma_{\mu \nu} U F^{\mu \nu} U^{\dagger}\right]$,

where $\mathrm{d} \sigma_{\mu \nu}$ is the surface measure on $\mathcal{S}$ and $U$ denotes a Wilson line connecting the point $x \in \mathcal{S}$ enclosed by the surface measure to an arbitrary base point $O$ on $\mathcal{C}$, which in Eq. (27) is taken to be $O \equiv\left(\infty, x_{1}\right)$.

\section{A local formulation for the dipole operator}

With similar considerations as before, let us establish yet another form for the dipole operator. This form allows for a power expansion where all terms are explicitly gauge invariant step by step. Starting from the gauge-rotated dipole in Eq. (19), for a rotation where gauge links are trivial at infinity, one has

$$
\mathcal{O}_{\xi}(\boldsymbol{b}, \boldsymbol{b}-\boldsymbol{r}) \rightarrow\left[U_{\boldsymbol{b}}(\infty, \xi) \Omega_{\boldsymbol{b}}(\xi)\right] \mathrm{e}^{-\boldsymbol{r} \cdot \partial_{b}}\left[\Omega_{\boldsymbol{b}}^{-1}(\xi) U_{\boldsymbol{b}}(\xi, \infty)\right],
$$

where we introduced the translation operator $\mathrm{e}^{-\boldsymbol{r} \cdot \partial_{b}}$. Without writing times for readability, let us note that

$$
\begin{aligned}
\partial_{b} \Omega^{-1} U & =\Omega^{-1} U\left[\partial_{b}+U^{\dagger} \Omega\left(\partial_{b} \Omega^{-1}\right) U+U^{\dagger}\left(\partial_{b} U\right)\right] \\
& =\Omega^{-1} U\left[\partial_{b}-i g \hat{\boldsymbol{A}}(\boldsymbol{b})\right],
\end{aligned}
$$

which leads to

$$
\left(\partial_{b}\right)^{n} \Omega^{-1} U^{\dagger}=\left(\partial_{b}-i g \hat{\boldsymbol{A}}(\boldsymbol{b})\right)^{n},
$$

where we can recognize the covariant derivative with the hatted gluon field from Eq. (25). With a simple exponentiation, we can now give two local forms for the dipole, in terms of the translation operator:

$$
\begin{aligned}
& (\mathcal{O}(\boldsymbol{b}, \boldsymbol{b}-\boldsymbol{r}))_{i j}=\left(\mathrm{e}^{-\boldsymbol{r} \cdot\left(\partial_{b}-i g \hat{\boldsymbol{A}}(\boldsymbol{b})\right)}\right)_{i j}, \\
& (\mathcal{O}(\boldsymbol{b}+\boldsymbol{r}, \boldsymbol{b}))_{i j}=\left(\mathrm{e}^{\boldsymbol{r} \cdot\left(\overline{\boldsymbol{\partial}}_{b}-i g \hat{\boldsymbol{A}}(\boldsymbol{b})\right)}\right)_{i j} .
\end{aligned}
$$

These forms allow for efficient and gauge-invariant power expansions, as discussed more explicitly in the Appendix.

\section{APPLICATION TO THE SMALL- $x$ AND TMD EQUIVALENCE FOR DIS DIJET PRODUCTION}

The amplitude for the production of a dijet in deep inelastic scattering (DIS), as obtained from the effective Feynman diagrams depicted in Fig. 2, involves the dipole operator. Starting from Eq. (26), one can apply successively relations (15) and then (17) in order to rewrite the dipole operator into one-body and two-body contributions:

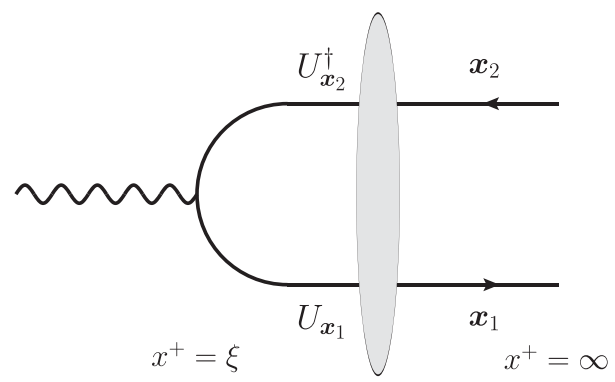

FIG. 2. The amplitude for DIS dijet production involves the nonsinglet dipole from Eq. (18). Gray blobs represent the interactions with the classical external field, which effectively dresses the quark and the antiquark with Wilson lines to build the dipole operator.

$$
\begin{aligned}
\mathcal{O}_{\xi}\left(\boldsymbol{x}_{1}, \boldsymbol{x}_{2}\right)= & 1-i g \int_{\boldsymbol{x}_{2}}^{x_{1}} \mathrm{~d} z^{i} \hat{\boldsymbol{A}}^{i}(\xi, \boldsymbol{z}) \\
& +(i g)^{2} \int_{\boldsymbol{x}_{2}}^{\boldsymbol{x}_{1}} \mathrm{~d} \boldsymbol{z}^{i} \int_{\boldsymbol{x}_{2}}^{z} \mathrm{~d} \boldsymbol{z}^{\prime j} \hat{\boldsymbol{A}}^{i}(\xi, \boldsymbol{z})\left[\hat{z}, \hat{z}^{\prime}\right] \hat{\boldsymbol{A}}^{j}\left(\xi, \boldsymbol{z}^{\prime}\right) .
\end{aligned}
$$

Then, recalling the explicit expression for the rotated fields in terms of twisted strength tensors,

$$
\begin{aligned}
\mathcal{O}_{\xi}\left(\boldsymbol{x}_{1}, \boldsymbol{x}_{2}\right)= & 1+i g \int_{\xi}^{\infty} \mathrm{d} t \int_{x_{2}}^{x_{1}} \mathrm{~d} z^{i} U_{z}(\infty, t) \\
& \times F^{-i}(t, z) U_{z}^{\dagger}(\infty, t) \\
& +(i g)^{2} \int_{\xi}^{\infty} \mathrm{d} t \int_{\xi}^{\infty} \mathrm{d} t^{\prime} \int_{x_{2}}^{x_{1}} \mathrm{~d} z^{i} \int_{x_{2}}^{z} \mathrm{~d} z^{\prime j} \\
& \times U_{z}(\infty, t) F^{-i}(t, z) U_{z}^{\dagger}(\infty, t)\left[\hat{z}, \hat{z}^{\prime}\right]_{t} \\
& \times U_{z^{\prime}}\left(\infty, t^{\prime}\right) F^{-j}\left(t^{\prime}, z^{\prime}\right) U_{z^{\prime}}^{\dagger}\left(\infty, t^{\prime}\right) .
\end{aligned}
$$

Using the expression for the hatted links

$$
\left[\hat{z}, \hat{z}^{\prime}\right]_{\xi}=U_{z}(\infty, \xi)\left[z, z^{\prime}\right]_{\xi} U_{z^{\prime}}^{\dagger}(\infty, \xi),
$$

as well as

$$
U_{z}^{\dagger}(\infty, t) U_{z}(\infty, \xi)=[t, \xi]_{z}
$$

and its counterpart for $\left[\xi, t^{\prime}\right]_{z^{\prime}}$, the dipole operator ends up entirely rewritten as the sum of one-body and two-body operators, in an explicitly gauge-invariant way:

$$
\begin{aligned}
\mathcal{O}_{\xi}\left(x_{1}, x_{2}\right)= & 1+i g \int_{\xi}^{\infty} \mathrm{d} t \int_{x_{2}}^{x_{1}} \mathrm{~d} z^{i}[\infty, t]_{z} F^{-i}(t, z)[t, \infty]_{z} \\
& +(i g)^{2} \int_{\xi}^{\infty} \mathrm{d} t \int_{\xi}^{\infty} \mathrm{d} t^{\prime} \int_{x_{2}}^{x_{1}} \mathrm{~d} z^{i} \int_{x_{2}}^{z} \mathrm{~d} z^{\prime j} \\
& \times[\infty, t]_{z} F^{-i}(t, z)[t, \xi]_{z}\left[z, z^{\prime}\right]_{\xi}\left[\xi, t^{\prime}\right]_{z^{\prime}} \\
& \times F^{-j}\left(t^{\prime}, z^{\prime}\right)\left[t^{\prime}, \infty\right]_{z^{\prime}}
\end{aligned}
$$


Equation (38) is very close to the result of Ref. [22] for the specific case of the dipole operator. With the simple trick for any function $F$

$$
F(z)=\int \frac{\mathrm{d}^{2} \boldsymbol{k}_{1}}{(2 \pi)^{2}} \int \mathrm{d}^{2} \boldsymbol{b}_{1} \mathrm{e}^{-i \boldsymbol{k}_{1} \cdot\left(\boldsymbol{b}_{1}-z\right)} F\left(\boldsymbol{b}_{1}\right)
$$

introducing $\boldsymbol{r} \equiv \boldsymbol{x}_{1}-\boldsymbol{x}_{2}$ and with straightforward algebra, we can finally recover that result:

$$
\begin{aligned}
\mathcal{O}_{\xi}\left(\boldsymbol{x}_{1}, \boldsymbol{x}_{2}\right)= & 1-i g \int_{\xi}^{\infty} \mathrm{d} t \int \frac{\mathrm{d}^{2} \boldsymbol{k}}{(2 \pi)^{2}} \boldsymbol{r}^{i}\left(\frac{\mathrm{e}^{i\left(\boldsymbol{k} \cdot \boldsymbol{x}_{1}\right)}-\mathrm{e}^{i\left(\boldsymbol{k} \cdot \boldsymbol{x}_{2}\right)}}{i(\boldsymbol{k} \cdot \boldsymbol{r})}\right) \\
& \times \int \mathrm{d}^{2} \boldsymbol{v} \mathrm{e}^{-i(\boldsymbol{k} \cdot \boldsymbol{v})}[\infty, t]_{\boldsymbol{v}} F^{i-}(t, \boldsymbol{v})[t, \infty]_{\boldsymbol{v}} \\
& +(i g)^{2} \int_{\xi}^{\infty} \mathrm{d} t \int_{\xi}^{\infty} \mathrm{d} t^{\prime} \int \frac{\mathrm{d}^{2} \boldsymbol{k}_{1}}{(2 \pi)^{2}} \int \frac{\mathrm{d}^{2} \boldsymbol{k}_{2}}{(2 \pi)^{2}} \int \mathrm{d}^{2} \boldsymbol{b}_{1} \int \mathrm{d}^{2} \boldsymbol{b}_{2} \mathrm{e}^{-i\left(\boldsymbol{k}_{1} \cdot \boldsymbol{b}_{1}\right)-i\left(\boldsymbol{k}_{2} \cdot \boldsymbol{b}_{2}\right)} \\
& \times \frac{\boldsymbol{r}^{i} \boldsymbol{r}^{j}}{i\left(\boldsymbol{k}_{2} \cdot \boldsymbol{r}\right)}\left(\frac{\mathrm{e}^{i\left(\boldsymbol{k}_{1}+\boldsymbol{k}_{2}\right) \cdot \boldsymbol{x}_{1}}-\mathrm{e}^{i\left(\boldsymbol{k}_{1}+\boldsymbol{k}_{2}\right) \cdot \boldsymbol{x}_{2}}}{i\left(\boldsymbol{k}_{1}+\boldsymbol{k}_{2}\right) \cdot \boldsymbol{r}}-\mathrm{e}^{i\left(\boldsymbol{k}_{2} \cdot \boldsymbol{x}_{2}\right)} \frac{\mathrm{e}^{i\left(\boldsymbol{k}_{1} \cdot \boldsymbol{x}_{1}\right)}-\mathrm{e}^{i\left(\boldsymbol{k}_{1} \cdot \boldsymbol{x}_{2}\right)}}{i\left(\boldsymbol{k}_{1} \cdot \boldsymbol{r}\right)}\right) \\
& \times[\infty, t]_{\boldsymbol{b}_{1}} F^{-i}\left(t, \boldsymbol{b}_{1}\right)[t, \xi]_{\boldsymbol{b}_{1}}\left[\boldsymbol{b}_{1}, \boldsymbol{b}_{2}\right]_{\xi}\left[\xi, t^{\prime}\right]_{\boldsymbol{b}_{2}} F^{-j}\left(t^{\prime}, \boldsymbol{b}_{2}\right)\left[t^{\prime}, \infty\right]_{\boldsymbol{b}_{2} \cdot}
\end{aligned}
$$

The one-body and two-body amplitudes that appear in the above decomposition of the dipole operator are depicted in Fig. 3.

This result can be used in order to rewrite any small- $x$ observable involving the dipole operator in terms of TMD distributions, as in Ref. [22]. Indeed, the expressions from Ref. [22] are straightforwardly recovered by convoluting the dipole operator $\mathcal{O}_{\xi}\left(\boldsymbol{x}_{1}, \boldsymbol{x}_{2}\right)$ with a hard part $\mathcal{H}\left(\boldsymbol{x}_{1}, \boldsymbol{x}_{2}\right)$ with its small- $x$ Galilean boost-invariant form $\mathcal{H}\left(\boldsymbol{x}_{1}, \boldsymbol{x}_{2}\right)=$ $e^{-i(\boldsymbol{\Delta} \cdot \boldsymbol{b})} \Phi(\boldsymbol{r})$, with $\boldsymbol{b}$ the impact parameter, $\boldsymbol{\Delta}$ the transverse momentum transfer, and $\Phi$ a wave function. Furthermore, the derivation presented above leads to an expression for TMD distributions which involves explicitly the previously neglected transverse gauge links which are usually assumed to be subdominant in light cone gauge in the small- $x$ regime. Here, we found an explicitly gauge-invariant expression for the operators. Still, note that the transverse gauge links involved here are built from pure gauge fields, and for full generality of this explicit gauge invariance one should consider the consequences of relaxing the assumption that transverse fields can be gauged away at small $x$. This is left for future studies.

It is interesting to note that the derivation of Eq. (40) is straightforward once the dipole operator is fully rewritten as the transverse string operator $\left[\hat{\boldsymbol{x}}_{1}, \hat{\boldsymbol{x}}_{2}\right]_{\xi}$, while working in the usual framework makes it quite technical. This representation also allows for a simple understanding of the structures encountered in Ref. [22]:

$$
\frac{\mathrm{e}^{i(\boldsymbol{k} \cdot \boldsymbol{r})}-1}{i(\boldsymbol{k} \cdot \boldsymbol{r})}
$$

as the Fourier transforms of identity along the contours which support the transverse strings. Namely,

$$
\int_{x_{1}}^{x_{2}} \mathrm{~d} z \mathrm{e}^{i z \cdot k}=r \int_{0}^{1} \mathrm{~d} s \mathrm{e}^{i s r \cdot k}
$$
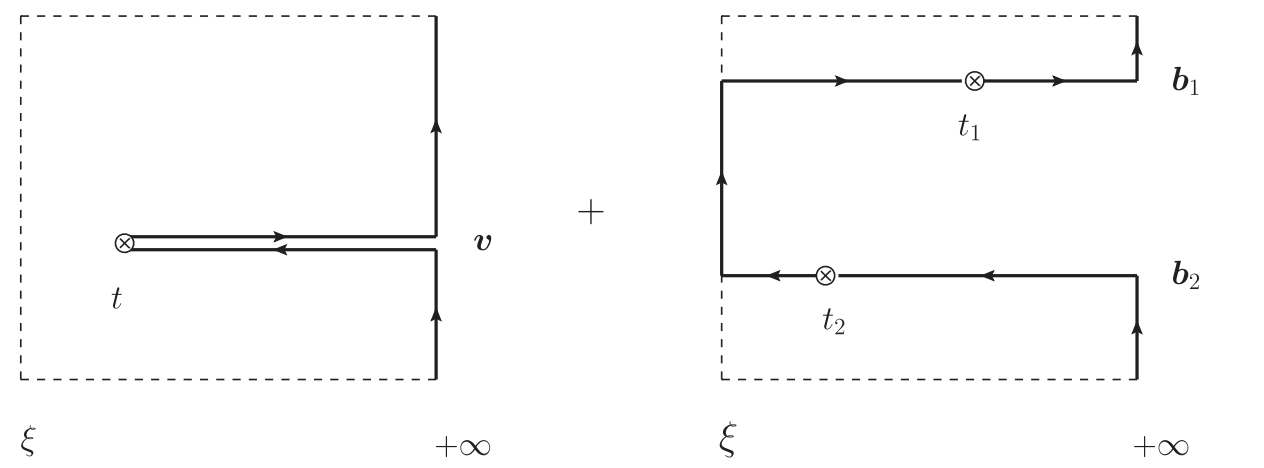

FIG. 3. Diagrammatic representation of the one-body (left) and two-body (right) amplitudes. 


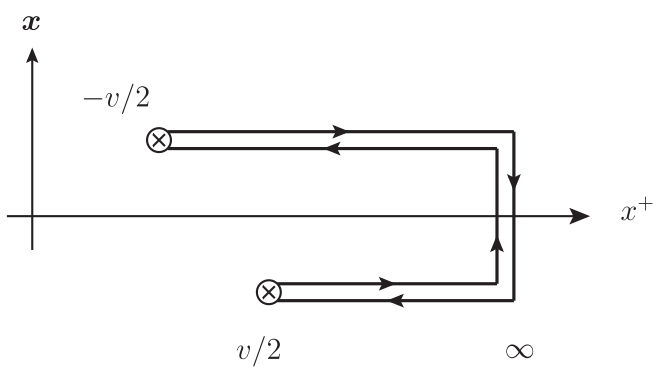

FIG. 4. Gauge link structure of the WW distribution.

Let us consider the leading genuine twist contribution to the dipole operator only:

$$
\begin{aligned}
\mathcal{O}_{\xi}\left(\boldsymbol{x}_{1}, \boldsymbol{x}_{2}\right)= & -i g \int_{\xi}^{\infty} \mathrm{d} t \int \frac{\mathrm{d}^{2} \boldsymbol{k}}{(2 \pi)^{2}} \boldsymbol{r}^{i}\left(\frac{\mathrm{e}^{i\left(\boldsymbol{k} \cdot \boldsymbol{x}_{1}\right)}-\mathrm{e}^{i\left(\boldsymbol{k} \cdot \boldsymbol{x}_{2}\right)}}{i(\boldsymbol{k} \cdot \boldsymbol{r})}\right) \\
& \times \int \mathrm{d}^{2} \boldsymbol{v} \mathrm{e}^{-i(\boldsymbol{k} \cdot \boldsymbol{v})}[\infty, t]_{\boldsymbol{v}} F^{i-}(t, \boldsymbol{v})[t, \infty]_{\boldsymbol{v}}
\end{aligned}
$$

and convolute it with the generic form of a $\gamma^{(*)} \rightarrow q \bar{q}$ hard part:

$\mathcal{H}\left(\boldsymbol{x}_{1}, \boldsymbol{x}_{2}\right)=(2 \pi) \delta(1-z-\bar{z}) \mathrm{e}^{-i\left(\boldsymbol{p}_{q} \cdot \boldsymbol{x}_{1}\right)-i\left(\boldsymbol{p}_{\bar{q}} \cdot \boldsymbol{x}_{2}\right)} \varphi(\boldsymbol{r})$,

where we used only the constraints from longitudinal momentum conservation through the classical field and the Galilean boost invariance of the photon wave function. We can easily obtain the $\mathcal{T}$ matrix for this process:

$$
\begin{aligned}
\mathcal{T}= & -g q^{+} \int \mathrm{d}^{2} \boldsymbol{r} \mathrm{e}^{-i\left(\bar{z} \boldsymbol{p}_{q}-z \boldsymbol{p}_{\bar{q}}\right) \cdot \boldsymbol{r}} \boldsymbol{r}^{i}\left(\frac{\mathrm{e}^{i \bar{z}\left(\boldsymbol{p}_{q}+\boldsymbol{p}_{\bar{q}}\right) \cdot \boldsymbol{r}}-\mathrm{e}^{-i z\left(\boldsymbol{p}_{q}+\boldsymbol{p}_{\bar{q}}\right) \cdot \boldsymbol{r}}}{i\left(\boldsymbol{p}_{q}+\boldsymbol{p}_{\bar{q}}\right) \cdot \boldsymbol{r}}\right) \\
& \times \varphi(\boldsymbol{r})[\infty, 0]_{\mathbf{0}} F^{i-}(0)[0, \infty]_{\mathbf{0}},
\end{aligned}
$$

which then leads to the following cross section:

$$
\begin{aligned}
\frac{d \sigma}{d z d^{2} \boldsymbol{p}_{q} d^{2} \boldsymbol{p}_{\bar{q}}}= & \int \frac{\mathrm{d}^{4} v}{(2 \pi)^{3}} \delta\left(v^{-}\right) \mathrm{e}^{-i\left(\boldsymbol{p}_{q}+\boldsymbol{p}_{\bar{q}}\right) \cdot \boldsymbol{v}}\left\langle P\left|F^{i-}\left(\frac{v}{2}\right) \mathcal{U}_{\bar{v},-\frac{v}{2}}^{[+]} F^{j-}\left(-\frac{v}{2}\right) \mathcal{U}_{-\frac{v}{2}, \frac{v}{2}}^{[+]}\right| P\right\rangle \\
& \times \frac{\alpha_{s} q^{+}}{8 z \bar{z} \pi s} \int \mathrm{d}^{2} \boldsymbol{r} \mathrm{d}^{2} \boldsymbol{r}^{\prime} \mathrm{e}^{-i\left(\bar{z} \boldsymbol{p}_{q}-z \boldsymbol{p}_{\bar{q}}\right) \cdot\left(\boldsymbol{r}-\boldsymbol{r}^{\prime}\right)} \boldsymbol{r}^{i} \boldsymbol{r}^{\prime j} \varphi(\boldsymbol{r}) \varphi^{*}\left(\boldsymbol{r}^{\prime}\right) \\
& \times\left(\frac{\mathrm{e}^{i \bar{z}\left(\boldsymbol{p}_{q}+\boldsymbol{p}_{\bar{q}}\right) \cdot \boldsymbol{r}}-\mathrm{e}^{-i z\left(\boldsymbol{p}_{q}+\boldsymbol{p}_{\bar{q}}\right) \cdot \boldsymbol{r}}}{\left(\boldsymbol{p}_{q}+\boldsymbol{p}_{\bar{q}}\right) \cdot \boldsymbol{r}}\right)\left(\frac{\mathrm{e}^{-i \bar{z}\left(\boldsymbol{p}_{q}+\boldsymbol{p}_{\bar{q}}\right) \cdot\left(\boldsymbol{r}^{\prime}\right)}-\mathrm{e}^{i z\left(\boldsymbol{p}_{q}+\boldsymbol{p}_{\bar{q}}\right) \cdot \boldsymbol{r}^{\prime}}}{\left(\boldsymbol{p}_{q}+\boldsymbol{p}_{\bar{q}}\right) \cdot \boldsymbol{r}^{\prime}}\right) .
\end{aligned}
$$

We clearly recognize the Weizsäcker-Williams TMD (1) from its gauge link structure, depicted in Fig. 4.

This cross section gives the form of the cross section for observables like dijet production in DIS if it was computed with the so-called small- $x$ improved TMD techniques [29], thus generalizing the equivalence found in Ref. [21] and extended in Ref. [22]. Note that the present results also contain genuine higher twist contributions, i.e., the $g^{2}$ terms in (40), which will not be displayed for the sake of readability. The reader is referred to Ref. [22] for more explicit genuine higher twist contributions, noting that the simple and explicitly gauge-invariant method we established in the present work allows for nonzero transverse gauge links in those contributions.

\section{EXTENSION TO GENERIC COLOR STRUCTURES}

Let us quickly extend the previous method for a more generic process: Let us consider a particle in color

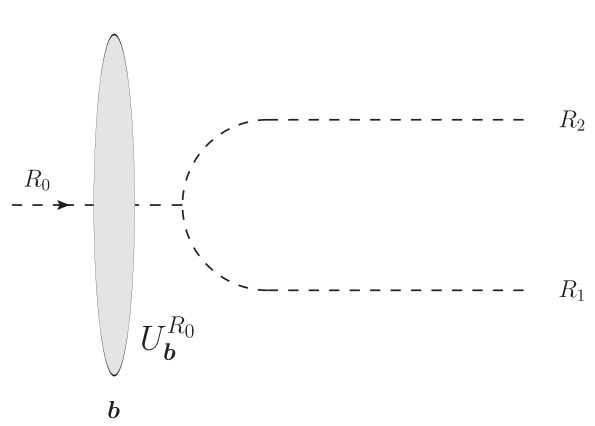

FIG. 5. Diagrams for the splitting of a particle in color representation $R_{0}$ into particles in respective representations $R_{1}$ and $R_{2}$, leading to the operator from Eq. (47). 
representation $R_{0}$ splitting into two particles in color representations $R_{1}$ and $R_{2}$ in the external classical field. The involved Wilson-line operator is then (see, e.g., [21])

$$
\begin{aligned}
\mathcal{O}_{\xi}^{012}\left(\boldsymbol{x}_{1}, \boldsymbol{x}_{2}\right)= & U_{\boldsymbol{x}_{1}}^{R_{1}}(\infty, \xi) T^{R_{0}} U_{\boldsymbol{x}_{2}}^{R_{2}}(\xi, \infty) \\
& -U_{\boldsymbol{b}}^{R_{1}}(\infty, \xi) T^{R_{0}} U_{\boldsymbol{b}}^{R_{2}}(\xi, \infty) .
\end{aligned}
$$

Here, $\boldsymbol{b}$ is the average position, weighted by longitudinal fractions $z$ and $\bar{z}: \boldsymbol{b}=z \boldsymbol{x}_{1}+\bar{z} \boldsymbol{x}_{2}$. This Wilson line structure is obtained by computing the diagrams in Fig. 5. We implicitly used the following identity, for open color indices in representations $R_{1}$ and in $R_{2}$ :

$$
T^{R_{0}} U_{b}^{R_{0}}=U_{b}^{R_{1}} T^{R_{0}} U_{b}^{R_{2}}
$$

As in the previous section, the transverse gauge links at infinity allow us to rewrite the operator with rotated Wilson lines:

$$
\begin{aligned}
\mathcal{O}_{\xi}^{012}\left(x_{1}, x_{2}\right) \rightarrow & \Omega_{x_{1}}^{R_{1} \dagger}(\infty) U_{x_{1}}^{R_{1}}(\infty, \xi) \Omega_{x_{1}}^{R_{1}}(\xi) T^{R_{0}} \Omega_{x_{2}}^{R_{2} \dagger}(\xi) U_{x_{2}}^{R_{2}}(\xi, \infty) \Omega_{x_{2}}^{R_{2}}(\infty) \\
& -\Omega_{x_{1}}^{R_{1} \dagger}(\infty) U_{b}^{R_{1}}(\infty, \xi) \Omega_{b}^{R_{1}}(\xi) T^{R_{0}} \Omega_{b}^{R_{2} \dagger}(\xi) U_{b}^{R_{2}}(\xi, \infty) \Omega_{x_{2}}^{R_{2}}(\infty) .
\end{aligned}
$$

As before, we will choose to cancel the gauge links at infinity. Here and from now on until the end of this section, we will omit the time dependence in the intermediate equations for the reader's convenience. The trick is now to write, for any $\boldsymbol{b}$,

$$
\Omega_{x_{1}}^{R_{1}} T^{R_{0}} \Omega_{x_{2}}^{R_{2} \dagger}=\Omega_{x_{1}}^{R_{1}} \Omega_{b}^{R_{1} \dagger} \Omega_{b}^{R_{1}} T^{R_{0}} \Omega_{b}^{R_{2} \dagger} \Omega_{b}^{R_{2}} \Omega_{x_{2}}^{R_{2} \dagger},
$$

then to interpret the $\Omega \Omega^{\dagger}$ pairs as transverse gauge links formed from the gauge-enhanced gluon fields [see Eqs. (16)]:

$$
\Omega_{x_{1}}^{R_{1}} T^{R_{0}} \Omega_{x_{2}}^{R_{2} \dagger}=\left[\boldsymbol{x}_{1}, \boldsymbol{b}\right]^{R_{1}} \Omega_{\boldsymbol{b}}^{R_{1}} T^{R_{0}} \Omega_{\boldsymbol{b}}^{R_{2} \dagger}\left[\boldsymbol{b}, \boldsymbol{x}_{2}\right]^{R_{2}},
$$

and finally to absorb the Wilson lines into rotated transverse links:

$$
U_{\boldsymbol{x}_{1}}^{R_{1}} \Omega_{x_{1}}^{R_{1}} T^{R_{0}} \Omega_{x_{2}}^{R_{2} \dagger} U_{\boldsymbol{x}_{2}}^{R_{2}}=\left[\hat{\boldsymbol{x}}_{1}, \hat{\boldsymbol{b}}\right]^{R_{1}} U_{\boldsymbol{b}}^{R_{1}} \Omega_{b}^{R_{1}} T^{R_{0}} \Omega_{\boldsymbol{b}}^{R_{2} \dagger} U_{\boldsymbol{b}}^{R_{2}}\left[\hat{\boldsymbol{b}}, \hat{\boldsymbol{x}}_{2}\right]^{R_{2}} .
$$

We can use Eqs. (15) and (17) once for each hatted link:

$$
\begin{aligned}
\mathcal{O}_{\xi}^{012}\left(\boldsymbol{x}_{1}, \boldsymbol{x}_{2}\right)= & -i g \int_{\boldsymbol{b}}^{x_{1}} \mathrm{~d} z^{i} \hat{\boldsymbol{A}}^{i R_{1}}(\boldsymbol{z})[\hat{\boldsymbol{z}}, \hat{\boldsymbol{b}}] U_{\boldsymbol{b}}^{R_{1}} \Omega_{\boldsymbol{b}}^{R_{1}} T^{R_{0}} \Omega_{\boldsymbol{b}}^{R_{2} \dagger} U_{\boldsymbol{b}}^{R_{2}}\left[\hat{\boldsymbol{b}}, \hat{\boldsymbol{x}}_{2}\right]^{R_{2}} \\
& -i g \int_{\boldsymbol{x}_{2}}^{\boldsymbol{b}} \mathrm{d} \boldsymbol{z}^{i} U_{\boldsymbol{b}}^{R_{1}} \Omega_{\boldsymbol{b}}^{R_{1}} T^{R_{0}} \Omega_{\boldsymbol{b}}^{R_{2} \dagger} U_{\boldsymbol{b}}^{R_{2}}[\hat{\boldsymbol{b}}, \hat{z}] \hat{\boldsymbol{A}}^{i R_{2}}(\boldsymbol{z}),
\end{aligned}
$$

or, equivalently,

$$
\begin{aligned}
\mathcal{O}_{\xi}^{012}\left(\boldsymbol{x}_{1}, \boldsymbol{x}_{2}\right)= & -i g \int_{\boldsymbol{b}}^{x_{1}} \mathrm{~d} z^{i} \hat{\boldsymbol{A}}^{i R_{1}}(\boldsymbol{z}) U_{z}^{R_{1}} \Omega_{z}^{R_{1}} T^{R_{0}} \Omega_{z}^{R_{2} \dagger} U_{z}^{R_{2}}\left[\hat{z}, \hat{\boldsymbol{x}}_{2}\right]^{R_{2}} \\
& -i g \int_{\boldsymbol{x}_{2}}^{\boldsymbol{b}} \mathrm{d} z^{\prime j}\left[\hat{\boldsymbol{b}}, \hat{z}^{\prime}\right]^{R_{1}} U_{z^{\prime}}^{R_{1}} \Omega_{z^{\prime}}^{R_{1}} T^{R_{0}} \Omega_{z^{\prime}}^{R_{2} \dagger} U_{z^{\prime}}^{R_{2}} \hat{\boldsymbol{A}}^{j R_{2}}\left(\boldsymbol{z}^{\prime}\right) .
\end{aligned}
$$

With similar tricks,

$$
\begin{aligned}
\mathcal{O}_{\xi}^{012}\left(x_{1}, x_{2}\right)= & -i g \int_{b}^{x_{1}} \mathrm{~d} z^{i} A^{i R_{1}}(z) U_{z}^{R_{1}} \Omega_{z}^{R_{1}} T^{R_{0}} \Omega_{z}^{R_{2} \dagger} U_{z}^{R_{2}} \\
& -i g \int_{x_{2}}^{b} \mathrm{~d} z^{i} U_{z}^{R_{1}} \Omega_{z}^{R_{1}} T^{R_{0}} \Omega_{z}^{R_{2} \dagger} U_{z}^{R_{2}} A^{i R_{2}}(z) \\
& +(i g)^{2} \int_{b}^{x_{1}} \mathrm{~d} z^{i} \int_{x_{2}}^{z} \mathrm{~d} z^{\prime j} A^{i R_{1}}(z) U_{z}^{R_{1}} \Omega_{z}^{R_{1}} T^{R_{0}} \Omega_{z}^{R_{2} \dagger}\left[\hat{z}, \hat{z}^{\prime}\right]^{R_{2}} U_{z^{\prime}}^{R_{2}} A^{j R_{2}}\left(z^{\prime}\right) \\
& +(i g)^{2} \int_{x_{2}}^{b} \mathrm{~d} z^{\prime j} \int_{z^{\prime}}^{b} \mathrm{~d} z^{i} A^{i R_{1}}(z) U_{z}^{R_{1}}\left[\hat{z}, \hat{z}^{\prime}\right]^{R_{1}} \Omega_{z^{\prime}}^{R_{1}} T^{R_{0}} \Omega_{z^{\prime}}^{R_{2} \dagger} U_{z^{\prime}}^{R_{2}} A^{j R_{2}}\left(z^{\prime}\right) .
\end{aligned}
$$


With $\Omega_{\infty}=1$, it is possible to replace $\Omega_{z}^{R_{1}}(\xi)$ by $[z, \infty]_{\xi}^{R_{1}}$ and $\Omega_{z^{\prime}}^{R^{\dagger}}(\xi)$ by $\left[\infty, z^{\prime}\right]_{\xi}^{R_{2}}$. Then using the definition of the (rotated) transverse fields and writing the time dependence explicitly again:

$$
\begin{aligned}
\mathcal{O}_{\xi}^{012}\left(x_{1}, x_{2}\right)= & -i g \int_{\xi}^{\infty} \mathrm{d} t \int_{b}^{x_{1}} \mathrm{~d} z^{i} U_{z}^{R_{1}}(\infty, t) T_{a}^{R_{1}} F_{a}^{i-}(t, z)[t, \xi]_{z}^{R_{1}}[z, \infty]_{\xi}^{R_{1}} T^{R_{0}}[\infty, z]_{\xi}^{R_{2}} U_{z}^{R_{2}}(\xi, \infty) \\
& -i g \int_{\xi}^{\infty} \mathrm{d} t \int_{x_{2}}^{b} \mathrm{~d} z^{i} U_{z}^{R_{1}}(\infty, \xi)[z, \infty]_{\xi}^{R_{1}} T^{R_{0}}[\infty, z]_{\xi}^{R_{2}}[\xi, t]_{z}^{R_{2}} T_{a}^{R_{2}} F_{a}^{i-}(t, z) U_{z}^{R_{2}}(t, \infty) \\
& +(i g)^{2} \int_{\xi}^{\infty} \mathrm{d} t \int_{\xi}^{\infty} \mathrm{d} t^{\prime}\left[\int_{b}^{x_{1}} \mathrm{~d} z^{i} \int_{x_{2}}^{z} \mathrm{~d} z^{\prime j}+\int_{x_{2}}^{b} \mathrm{~d} z^{\prime j} \int_{z^{\prime}}^{b} \mathrm{~d} z^{i}\right] \\
& \times U_{z}^{R_{1}}(\infty, t) T_{a}^{R_{1}} F_{a}^{i-}(t, z)[t, \xi]_{z}^{R_{1}}[z, \infty]_{\xi}^{R_{1}} T^{R_{0}}\left[\infty, z^{\prime}\right]_{\xi}^{R_{2}}\left[\xi, t^{\prime}\right]_{z^{\prime}}^{R_{2}} T_{b}^{R_{2}} F_{b}^{j-}\left(t^{\prime}, z^{\prime}\right) U_{z^{\prime}}^{R_{2}}\left(t^{\prime}, \infty\right) .
\end{aligned}
$$

With the use of the trick given in Eq. (39), one recovers the results from Ref. [22], with explicit transverse gauge links:

$$
\begin{aligned}
\mathcal{O}_{\xi}^{012}\left(\boldsymbol{x}_{1}, \boldsymbol{x}_{2}\right)= & -i g \bar{z} \boldsymbol{r}^{i} \int_{\xi}^{\infty} \mathrm{d} t \int \frac{\mathrm{d}^{2} \boldsymbol{k}}{(2 \pi)^{2}} \int \mathrm{d}^{2} \boldsymbol{v} \mathrm{e}^{-i \boldsymbol{k} \cdot\left(\boldsymbol{v}-\boldsymbol{x}_{1}\right)} \frac{\mathrm{e}^{i \bar{z}(\boldsymbol{k} \cdot \boldsymbol{r})}-1}{i \bar{z}(\boldsymbol{k} \cdot \boldsymbol{r})} \\
& \times[\infty, t]_{v}^{R_{1}} T_{a}^{R_{1}} F_{a}^{i-}(t, \boldsymbol{v})[t, \xi]_{\boldsymbol{v}}^{R_{1}}[\boldsymbol{v}, \infty]_{\xi}^{R_{1}} T^{R_{0}}[\boldsymbol{\infty}, \boldsymbol{v}]_{\xi}^{R_{2}}[\xi, \infty]_{\boldsymbol{v}}^{R_{2}} \\
& -i g z \boldsymbol{r}^{i} \int_{\xi}^{\infty} \mathrm{d} t \int \frac{\mathrm{d}^{2} \boldsymbol{k}}{(2 \pi)^{2}} \int \mathrm{d}^{2} \boldsymbol{v} \mathrm{e}^{-i \boldsymbol{k} \cdot\left(\boldsymbol{v}-\boldsymbol{x}_{2}\right)} \frac{\mathrm{e}^{i z(\boldsymbol{k} \cdot \boldsymbol{r})}-1}{i z(\boldsymbol{k} \cdot \boldsymbol{r})} \\
& \times[\infty, \xi]_{\boldsymbol{v}}^{R_{1}}[\boldsymbol{v}, \infty]_{\xi}^{R_{1}} T^{R_{0}}[\infty, \boldsymbol{v}]_{\xi}^{R_{2}}[\xi, t]_{v}^{R_{2}} T_{a}^{R_{2}} F_{a}^{i-}(t, \boldsymbol{v})[t, \infty]_{\boldsymbol{v}}^{R_{2}} \\
& +(i g)^{2} \int_{\xi}^{\infty} \mathrm{d} t \int_{\xi}^{\infty} \mathrm{d} t^{\prime} \int \frac{\mathrm{d}^{2} \boldsymbol{k}_{1}}{(2 \pi)^{2}} \int \frac{\mathrm{d}^{2} \boldsymbol{k}_{2}}{(2 \pi)^{2}} \int \mathrm{d}^{2} \boldsymbol{b}_{1} \int \mathrm{d}^{2} \boldsymbol{b}_{2} \mathrm{e}^{-i \boldsymbol{k}_{1} \cdot\left(\boldsymbol{b}_{1}-\boldsymbol{b}\right)} \mathrm{e}^{-i \boldsymbol{k}_{2} \cdot\left(\boldsymbol{b}_{2}-\boldsymbol{b}\right)} \\
& \times \frac{\boldsymbol{r}^{i} \boldsymbol{r}^{j}}{\left(\boldsymbol{k}_{1}+\boldsymbol{k}_{2}\right) \cdot \boldsymbol{r}}\left(\frac{\mathrm{e}^{-i\left(\boldsymbol{k}_{2} \cdot \boldsymbol{r}\right)}-1}{\left(\boldsymbol{k}_{2} \cdot \boldsymbol{r}\right)} \mathrm{e}^{i \bar{z}\left(\boldsymbol{k}_{1}+\boldsymbol{k}_{2}\right) \cdot \boldsymbol{r}}+\frac{\mathrm{e}^{i\left(\boldsymbol{k}_{1} \cdot \boldsymbol{r}\right)}-1}{\left(\boldsymbol{k}_{1} \cdot \boldsymbol{r}\right)} \mathrm{e}^{-i z\left(\boldsymbol{k}_{1}+\boldsymbol{k}_{2}\right) \cdot \boldsymbol{r}}\right) \\
& \times[\infty, t]_{\boldsymbol{b}_{1}}^{R_{1}} T_{a}^{R_{1}} F_{a}^{i-}\left(t, \boldsymbol{b}_{1}\right)[t, \xi]_{\boldsymbol{b}_{1}}^{R_{1}}\left[\boldsymbol{b}_{1}, \infty\right]_{\xi}^{R_{1}} T^{R_{0}}\left[\infty, \boldsymbol{b}_{2}\right]_{\xi}^{R_{2}}\left[\xi, t^{\prime}\right]_{\boldsymbol{b}_{2}}^{R_{2}} T_{b}^{R_{2}} F_{b}^{j-}\left(t^{\prime}, \boldsymbol{b}_{2}\right)\left[t^{\prime}, \infty\right]_{\boldsymbol{b}_{2}}^{R_{2}} .
\end{aligned}
$$

\section{EXTENSION TO THREE-LINE OPERATORS}

The formulation of Wilson-line operators in terms of transverse gauge links makes it very easy to extend the proof of equivalence to TMD distributions for observables with more than two Wilson lines involved. For example, let us consider the $\gamma^{(*)} \rightarrow q \bar{q} g$ amplitude. It involves operators with two Wilson lines, which can be treated as in previous sections, but it also contains a three-line operator [30]

$$
\mathcal{M}_{3}=U_{x_{1}} \mathbf{t}^{b} W_{x_{3}}^{b a} U_{x_{2}}^{\dagger},
$$

which in the fundamental representation reads as a four-line one:

$$
\mathcal{M}_{3}=U_{x_{1}} U_{x_{3}}^{\dagger} \mathbf{t}^{a} U_{x_{3}} U_{x_{2}}^{\dagger} .
$$

Taking into account all three factors $\Omega(\infty)$ and using the tricks from Sec. III, this operator becomes

$$
\mathcal{M}_{3}=\left[\hat{\boldsymbol{x}}_{1}, \hat{\boldsymbol{x}}_{3}\right]_{\xi} \mathbf{t}^{a}\left[\hat{\boldsymbol{x}}_{3}, \hat{\boldsymbol{x}}_{2}\right]_{\xi} .
$$

Applying Eq. (34) for $\xi=-\infty$ simultaneously on the left and on the right of the color matrix and using the explicit expressions for the rotated fields (25) allows one to rewrite directly $\mathcal{U}_{3}$ with one-, two-, three-, and four-body contributions, reading, respectively [31],

$$
\begin{aligned}
\mathcal{M}_{3}^{(1)}= & -i g \int \mathrm{d} t \int_{x_{3}}^{x_{1}} \mathrm{~d} z^{i}[\infty, t]_{z} F^{i-}(t, z)[t, \infty]_{z} t^{b} \\
& -i g \int \mathrm{d} t \int_{x_{2}}^{x_{3}} \mathrm{~d} z^{i} \mathbf{t}^{b}[\infty, t]_{z} F^{i-}(t, z)[t, \infty]_{z}, \quad(61) \\
\mathcal{U}_{3}^{(2)}= & (i g)^{2} \int \mathrm{d} t \int \mathrm{d} t^{\prime} \int_{x_{3}}^{x_{1}} \mathrm{~d} z^{i} \int_{x_{3}}^{z} \mathrm{~d} z^{\prime j} \\
& \times[\infty, t]_{z} F^{i-}(t, z)[t, \xi]_{z}\left[z, z^{\prime}\right]_{\xi}\left[\xi, t^{\prime}\right]_{z^{\prime}} F^{j-}\left(t^{\prime}, z^{\prime}\right)\left[t^{\prime}, \infty\right]_{z^{\prime}} \mathbf{t}^{b} \\
& +(i g)^{2} \int \mathrm{d} t \int \mathrm{d} t^{\prime} \int_{x_{3}}^{x_{1}} \mathrm{~d} z^{i} \int_{x_{2}}^{x_{3}} \mathrm{~d} z^{\prime j} \\
& \times[\infty, t]_{z} F^{i-}(t, z)[t, \infty]_{z} t^{b}\left[\infty, t^{\prime}\right]_{z^{\prime}} F^{j-}\left(t^{\prime}, z^{\prime}\right)\left[t^{\prime}, \infty\right]_{z^{\prime}} \\
& +(i g)^{2} \int \mathrm{d} t \int \mathrm{d} t^{\prime} \int_{x_{2}}^{x_{3}} \mathrm{~d} z^{i} \int_{x_{2}}^{z} \mathrm{~d} z^{\prime j} \\
& \times \mathbf{t}^{b}[\infty, t]_{z} F^{i-}(t, z)[t, \xi]_{z}\left[z, z^{\prime}\right]_{\xi}\left[\xi, t^{\prime}\right]_{z^{\prime}} F^{j-}\left(t^{\prime}, z^{\prime}\right)\left[t^{\prime}, \infty\right]_{z^{\prime}},
\end{aligned}
$$




$$
\begin{aligned}
\mathcal{U}_{3}^{(3)}= & -(i g)^{3} \int \mathrm{d} t_{1} \int \mathrm{d} t_{2} \int \mathrm{d} t_{3} \int_{x_{3}}^{x_{1}} \mathrm{~d} z^{i} \int_{\boldsymbol{x}_{3}}^{z} \mathrm{~d} z^{\prime j} \int_{\boldsymbol{x}_{2}}^{x_{3}} \mathrm{~d} \boldsymbol{u}^{k} \\
& \times\left[\infty, t_{1}\right]_{z} F^{i-}\left(t_{1}, z\right)\left[t_{1}, \xi\right]_{z}\left[z, z^{\prime}\right]_{\xi}\left[\xi, t_{2}\right]_{z^{\prime}} F^{j-}\left(t_{2}, \boldsymbol{z}^{\prime}\right) \\
& \times\left[t_{2}, \infty\right]_{z^{\prime}} \mathbf{t}^{b}\left[\infty, t_{3}\right]_{\boldsymbol{u}} F^{k-}\left(t_{3}, \boldsymbol{u}\right)\left[t_{3}, \infty\right]_{\boldsymbol{u}} \\
& -(i g)^{3} \int \mathrm{d} t_{1} \int \mathrm{d} t_{2} \int \mathrm{d} t_{3} \int_{\boldsymbol{x}_{3}}^{x_{1}} \mathrm{~d} z^{i} \int_{\boldsymbol{x}_{2}}^{x_{3}} \mathrm{~d} \boldsymbol{u}^{j} \int_{\boldsymbol{x}_{2}}^{\boldsymbol{u}} \mathrm{d} \boldsymbol{u}^{\prime k} \\
& \times\left[\infty, t_{1}\right]_{z} F^{i-}\left(t_{1}, z\right)\left[t_{1}, \infty\right]_{z} \mathbf{z}^{b}\left[\infty, t_{2}\right]_{\boldsymbol{u}} F^{j-}\left(t_{2}, \boldsymbol{u}\right) \\
& \times\left[t_{2}, \xi\right]_{\boldsymbol{u}}\left[\boldsymbol{u}, \boldsymbol{u}^{\prime}\right]_{\xi}\left[\xi, t_{3}\right]_{\boldsymbol{u}^{\prime}} F^{k-}\left(t_{3}, \boldsymbol{u}^{\prime}\right)\left[t_{3}, \infty\right]_{\boldsymbol{u}^{\prime}},
\end{aligned}
$$

and

$$
\begin{aligned}
\mathcal{U}_{3}^{(4)}= & (i g)^{4} \int d t_{1} \int \mathrm{d} t_{2} \int \mathrm{d} t_{3} \int \mathrm{d} t_{4} \int_{\boldsymbol{x}_{3}}^{x_{1}} \mathrm{~d} z^{i} \int_{\boldsymbol{x}_{3}}^{z} \mathrm{~d} z^{\prime j} \\
& \times \int_{\boldsymbol{x}_{2}}^{x_{3}} \mathrm{~d} \boldsymbol{u}^{k} \int_{\boldsymbol{x}_{2}}^{\boldsymbol{u}} \mathrm{d} \boldsymbol{u}^{\prime l}\left[\boldsymbol{\infty}, t_{1}\right]_{z} F^{i-}\left(t_{1}, \boldsymbol{z}\right)\left[t_{1}, \xi\right]_{z}\left[z, \boldsymbol{z}^{\prime}\right]_{\xi} \\
& \times\left[\xi, t_{2}\right]_{z^{\prime}} F^{j-}\left(t_{2}, \boldsymbol{z}^{\prime}\right)\left[t_{2}, \infty\right]_{z^{\prime}} \mathbf{t}^{b}\left[\boldsymbol{\infty}, t_{3}\right]_{\boldsymbol{u}} F^{k-}\left(t_{3}, \boldsymbol{u}\right) \\
& \times\left[t_{3}, \xi\right]_{\boldsymbol{u}}\left[\boldsymbol{u}, \boldsymbol{u}^{\prime}\right]_{\xi}\left[\xi, t_{4}\right]_{\boldsymbol{u}^{\prime}} F^{l-}\left(t_{4}, \boldsymbol{u}^{\prime}\right)\left[t_{4}, \infty\right]_{\boldsymbol{u}^{\prime}} \cdot
\end{aligned}
$$

Although the final expressions are quite cumbersome, the method to derive them is straightforward once the dipoles have been replaced by transverse strings. All it took was Eq. (34).

\section{DISCUSSIONS}

We have provided a reinterpretation of operators built from Wilson lines as operators built from transverse strings. This observation allows for compact and systematic extensions of the exact small- $x$ and TMD equivalence shown in Ref. [22]. It also allows for a systematic power expansion which preserves QCD gauge invariance at each step. We have given the example of an extension beyond previously established results.

Besides the fact that small- $x$ models can lead to interesting insight on TMD distributions at asymptotic energies, the infinite-twist TMD form of small- $x$ amplitudes also changes our understanding of saturation effects in the dilute-dense regime [22] by distinguishing between kinematic and genuine saturation effects. In light of this new development, it would be interesting to revisit the nonlinear terms in small- $x$ evolution in terms of TMD distributions. The first step toward the understanding of these nonlinearities is precisely what we accomplished in our example from Sec. VI, by extracting the TMD operators from the three-line operator which appears when one evolves a dipole.

Another advantage of the TMD form of small- $x$ amplitudes is the opportunity it opens for the use of TMD evolution equations to resum logarithms of the hard scale as well as Sudakov logarithms. Thanks to the extension described in Sec. VI, it is now possible to adapt the full next-to-leading logarithmic cross section for inclusive DIS $[32,33]$ in order to include these resummations.

\section{ACKNOWLEDGMENTS}

This work is supported by the U.S. Department of Energy, Office of Science, Office of Nuclear Physics, under Contract No. DE-SC0012704, and in part by Laboratory Directed Research and Development (LDRD) funds from Brookhaven Science Associates. R. B. is supported by the Los Alamos National Laboratory Directed Research and Development program.

\section{APPENDIX: GAUGE-INVARIANT POWER EXPANSION: LOCAL FORMULATION}

In this Appendix, we shall use the formulation of the dipole operator established above in order to construct a power expansion of the dipole operator Eq. (26) that is explicitly gauge invariant order by order in powers of $\boldsymbol{r}=\boldsymbol{x}_{2}-\boldsymbol{x}_{1}$. Equations (32) and (33) can be used in order to perform a local expansion, thus an expansion in powers of small transverse momenta, with explicit gauge invariance of all operators at each step of the process. For this purpose, one can use the following identity which is a direct consequence of Eq. (21) [34]:

$$
\partial_{\boldsymbol{b}}^{i}-\hat{A}^{i}\left(t_{0}, \boldsymbol{b}\right)=U_{\boldsymbol{b}}\left(\infty, t_{0}\right) D^{i}\left(t_{0}, \boldsymbol{b}\right) U_{\boldsymbol{b}}^{\dagger}\left(t_{0}, \infty\right) .
$$

Let us detail the steps for the second term of the local expansion of Eq. (32). First, use the definition of the hatted field on the right:

$$
\begin{aligned}
\mathcal{O}^{(2)}(\boldsymbol{b}, \boldsymbol{b}-\boldsymbol{r}) & =\boldsymbol{r}^{i_{1}} \boldsymbol{r}^{i_{0}}\left(\partial_{\boldsymbol{b}}-i g \hat{\boldsymbol{A}}(-\infty, \boldsymbol{b})\right)^{i_{1}}\left(\partial_{\boldsymbol{b}}-i g \hat{\boldsymbol{A}}(-\infty, \boldsymbol{b})\right)^{i_{0}} \\
& =-i g \int_{-\infty}^{+\infty} \mathrm{d} t_{0} \boldsymbol{r}^{i_{1}} \boldsymbol{r}^{i_{0}}\left(\partial_{\boldsymbol{b}}-i g \hat{\boldsymbol{A}}(-\infty, \boldsymbol{b})\right)^{i_{1}}\left[U_{\boldsymbol{b}}\left(\infty, t_{0}\right) F^{i_{0}-}\left(t_{0}, \boldsymbol{b}\right) U_{\boldsymbol{b}}^{\dagger}\left(t_{0}, \infty\right)\right] .
\end{aligned}
$$

The trick is now to write the hatted field on the left, which is evaluated at $-\infty$ light cone time, as a function of the hatted field at light cone time $t_{0}$. Thus, we need to use the following relation [cf. Eq. (25)]:

$$
\hat{A}^{i}(-\infty, \boldsymbol{b}) \equiv \hat{A}^{i}\left(t_{0}, \boldsymbol{b}\right)+\int_{-\infty}^{t_{0}} \mathrm{~d} t U(+\infty, t) F^{i-}(t, \boldsymbol{b}) U^{\dagger}(t,+\infty),
$$


which, when combined with Eq. (A1), leads to

$$
\begin{aligned}
\left(\partial_{\boldsymbol{b}}-i g \hat{\boldsymbol{A}}(-\infty, \boldsymbol{b})\right)^{i_{1}} & =\partial_{\boldsymbol{b}}^{i_{1}}-i g \hat{\boldsymbol{A}}^{i_{1}}\left(t_{0}, \boldsymbol{b}\right)-i g \int_{-\infty}^{t_{0}} \mathrm{~d} t_{1} U_{\boldsymbol{b}}\left(\infty, t_{1}\right) F^{i_{1}-}\left(t_{1}, \boldsymbol{b}\right) U_{\boldsymbol{b}}^{\dagger}\left(t_{1}, \infty\right) \\
& =U_{\boldsymbol{b}}\left(\infty, t_{0}\right) D^{i_{1}}\left(t_{0}, \boldsymbol{b}\right) U_{\boldsymbol{b}}^{\dagger}\left(t_{0}, \infty\right)-i g \int_{-\infty}^{t_{0}} \mathrm{~d} t_{1} U_{\boldsymbol{b}}\left(\infty, t_{1}\right) F^{i_{1}-}\left(t_{1}, \boldsymbol{b}\right) U_{\boldsymbol{b}}^{\dagger}\left(t_{1}, \infty\right) .
\end{aligned}
$$

We can then conclude with

$$
\begin{aligned}
\mathcal{O}_{2}= & -i g \boldsymbol{r}^{i_{1}} \boldsymbol{r}^{i_{0}} \int_{-\infty}^{+\infty} \mathrm{d} t_{0}\left[\infty, t_{0}\right]_{\boldsymbol{b}} D^{i_{1}}\left(t_{0}, \boldsymbol{b}\right)\left(F^{i_{0}-}\left(t_{0}, \boldsymbol{b}\right)\left[t_{0}, \infty\right]_{\boldsymbol{b}}\right) \\
& -g^{2} \boldsymbol{r}^{i_{1}} \boldsymbol{r}^{i_{0}} \int_{-\infty}^{+\infty} \mathrm{d} t_{0} \int_{-\infty}^{t_{0}} \mathrm{~d} t_{1}\left[\infty, t_{1}\right]_{\boldsymbol{b}} F^{i_{1}-}\left(t_{1}, \boldsymbol{b}\right)\left[t_{1}, t_{0}\right]_{\boldsymbol{b}} F^{i_{0}-}\left(t_{0}, \boldsymbol{b}\right)\left[t_{0}, \infty\right]_{\boldsymbol{b}} .
\end{aligned}
$$

By recursion with similar steps, one can prove the general form of the $|\boldsymbol{r}|^{n}$ term:

$$
\begin{aligned}
\mathcal{O}_{n}= & (-1)^{n} \boldsymbol{r}^{i_{m}} \ldots \boldsymbol{r}^{i_{1}} \boldsymbol{r}^{i_{0}} \sum_{k_{0} \ldots k_{m}} \int_{-\infty}^{\infty} \mathrm{d} t_{0} \int_{-\infty}^{t_{0}} \mathrm{~d} t_{1} \ldots \int_{-\infty}^{t_{m-1}} \mathrm{~d} t_{m} \\
& \times U\left(+\infty, t_{m}\right)(\boldsymbol{r} \cdot D)^{k_{m}} F^{i_{m}-}\left(t_{m}\right) U^{\dagger}\left(t_{m}, t_{m-1}\right) \ldots U^{\dagger}\left(t_{1}, t_{0}\right)(\boldsymbol{r} \cdot D)^{k_{0}} F^{i_{0}-}\left(t_{0}\right) U^{\dagger}\left(t_{0},+\infty\right),
\end{aligned}
$$

where the sum over $k_{0} \ldots k_{m}$ is constrained by

$$
\sum_{j=0} k_{j}=n-m
$$

In simple words, the $n$th term in the gauge-invariant local expansion of the dipole operator is the sum of all possible insertions of $F$ tensors and covariant derivatives with the appropriate gauge links, such that the number of $F$ 's and the number of $D$ 's sum up to $n$. As an illustration, the first few orders read

$$
\mathcal{O}_{1}^{i}=-\boldsymbol{r}^{i} \int_{-\infty}^{+\infty} \mathrm{d} t[+\infty, t] F^{i-}(t)[t,+\infty]
$$

for the first order,

$$
\begin{aligned}
\mathcal{O}_{2}^{i j}= & \boldsymbol{r}^{i} \boldsymbol{r}^{j} \int_{-\infty}^{+\infty} \mathrm{d} t_{1} \int_{-\infty}^{t_{1}} \mathrm{~d} t_{2}\left[+\infty, t_{2}\right] F^{i-}\left[t_{2}, t_{1}\right] F^{j-}\left[t_{1},+\infty\right] \\
& +\int_{-\infty}^{+\infty} \mathrm{d} t[+\infty, t] D^{j} F^{i-}(t)[t,+\infty]
\end{aligned}
$$

for the second, and for the third we have

$$
\begin{aligned}
\mathcal{O}_{3}^{i j k}= & -\boldsymbol{r}^{i} \boldsymbol{r}^{j} \boldsymbol{r}^{k} \int_{-\infty}^{+\infty} \mathrm{d} t_{1} \int_{-\infty}^{t_{1}} \mathrm{~d} t_{2} \int_{-\infty}^{t_{2}} \mathrm{~d} t_{3}\left[\infty, t_{3}\right] F^{i-}\left[t_{3}, t_{2}\right] F^{j-}\left[t_{3}, t_{2}\right] F^{k-}\left[t_{1},+\infty\right] \\
& +\int_{-\infty}^{+\infty} \mathrm{d} t_{1} \int_{-\infty}^{t_{1}} \mathrm{~d} t_{2}\left[+\infty, t_{2}\right]\left(D^{k} F^{i-}\left[t_{2}, t_{1}\right] F^{j-}+F^{i-}\left[t_{2}, t_{1}\right] D^{k} F^{j-}\right)\left[t_{1},+\infty\right] \\
& +\int_{-\infty}^{\infty} \mathrm{d} t[+\infty, t] D^{j} D^{k} F^{i-}(t)[t,+\infty]
\end{aligned}
$$

The formulation of the dipole operator as a transverse QCD string can thus be used in order to perform the power expansion of a small- $x$ observable while keeping explicitly gauge-invariant operators. This is a particularly difficult task when using more standard forms of the small $x$ observables, where the gluon field strength tensor appears only via the derivative of Wilson lines thanks to the relation $\partial^{i} A^{-}=F^{i-}$. For example, see Ref. [13], where gauge invariance, while not broken, is not explicitly respected due to the presence of double derivatives of Wilson lines which lead to simple derivatives of fields. Here, we established a systematic framework to perform such expansions with explicit invariance. 
[1] L. D. McLerran and R. Venugopalan, Computing quark and gluon distribution functions for very large nuclei, Phys. Rev. D 49, 2233 (1994).

[2] L. D. McLerran and R. Venugopalan, Gluon distribution functions for very large nuclei at small transverse momentum, Phys. Rev. D 49, 3352 (1994).

[3] L. D. McLerran and R. Venugopalan, Green's functions in the color field of a large nucleus, Phys. Rev. D 50, 2225 (1994).

[4] I. Balitsky, Operator expansion for high-energy scattering, Nucl. Phys. B463, 99 (1996).

[5] I. Balitsky, Factorization for High-Energy Scattering, Phys. Rev. Lett. 81, 2024 (1998).

[6] I. Balitsky, Factorization and high-energy effective action, Phys. Rev. D 60, 014020 (1999).

[7] F. Dominguez, C. Marquet, B.-W. Xiao, and F. Yuan, Universality of unintegrated gluon distributions at small $x$, Phys. Rev. D 83, 105005 (2011).

[8] Bold symbols denote the transverse components of fourvectors in Euclidean space.

[9] F. Dominguez, B.-W. Xiao, and F. Yuan, $k_{t}$-Factorization for Hard Processes in Nuclei, Phys. Rev. Lett. 106, 022301 (2011).

[10] In this work, we adopt the standard notation $\partial^{\mu} \equiv \partial / \partial x_{\mu}$ for the partial derivatives.

[11] A. Metz and J. Zhou, Distribution of linearly polarized gluons inside a large nucleus, Phys. Rev. D 84, 051503 (2011).

[12] E. Akcakaya, A. Schäfer, and J. Zhou, Azimuthal asymmetries for quark pair production in $\mathrm{pA}$ collisions, Phys. Rev. D 87, 054010 (2013).

[13] A. Dumitru and V. Skokov, $\cos (4 \varphi)$ azimuthal anisotropy in small- $x$ DIS dijet production beyond the leading power TMD limit, Phys. Rev. D 94, 014030 (2016).

[14] C. Marquet, E. Petreska, and C. Roiesnel, Transversemomentum-dependent gluon distributions from JIMWLK evolution, J. High Energy Phys. 10 (2016) 065.

[15] D. Boer, P. J. Mulders, J. Zhou, and Y.-j. Zhou, Suppression of maximal linear gluon polarization in angular asymmetries, J. High Energy Phys. 10 (2017) 196.

[16] C. Marquet, C. Roiesnel, and P. Taels, Linearly polarized small- $x$ gluons in forward heavy-quark pair production, Phys. Rev. D 97, 014004 (2018).

[17] E. Petreska, TMD gluon distributions at small $x$ in the CGC theory, Int. J. Mod. Phys. E 27, 1830003 (2018).

[18] T. Altinoluk, R. Boussarie, C. Marquet, and P. Taels, TMD factorization for dijets + photon production from the dilute-dense CGC framework, J. High Energy Phys. 07 (2019) 079.
[19] I. Balitsky and A. Tarasov, Rapidity evolution of gluon TMD from low to moderate $\mathrm{x}$, J. High Energy Phys. 10 (2015) 017.

[20] I. Balitsky and G. A. Chirilli, Conformal invariance of transverse-momentum dependent parton distributions rapidity evolution, Phys. Rev. D 100, 051504 (2019).

[21] T. Altinoluk, R. Boussarie, and P. Kotko, Interplay of the CGC and TMD frameworks to all orders in kinematic twist, J. High Energy Phys. 05 (2019) 156.

[22] T. Altinoluk and R. Boussarie, Low $x$ physics as an infinite twist (G)TMD framework: Unravelling the origins of saturation, J. High Energy Phys. 10 (2019) 208.

[23] F. Gelis, E. Iancu, J. Jalilian-Marian, and R. Venugopalan, The color glass condensate, Annu. Rev. Nucl. Part. Sci. 60, 463 (2010).

[24] G. A. Chirilli, Y. V. Kovchegov, and D. E. Wertepny, Regularization of the light-cone gauge gluon propagator singularities using sub-gauge conditions, J. High Energy Phys. 12 (2015) 138.

[25] From now on, we will denote light cone times with standard time notations $\xi$ or $t$ for the reader's convenience.

[26] Note that $U_{x}^{\dagger}(t, \xi)=U_{x}(\xi, t)$ owing to the fact that the classical gauge field is real. In this article, we will choose to emphasize explicit continuity of light cone time ordering in the operators by using the rhs, while in most small- $x$ literature the lhs is chosen.

[27] P. M. Fishbane, S. Gasiorowicz, and P. Kaus, Stokes' theorems for non-Abelian fields, Phys. Rev. D 24, 2324 (1981).

[28] U. A. Wiedemann, Transverse dynamics of hard partons in nuclear media and the QCD dipole, Nucl. Phys. B582, 409 (2000).

[29] P. Kotko, K. Kutak, C. Marquet, E. Petreska, S. Sapeta, and A. van Hameren, Improved TMD factorization for forward dijet production in dilute-dense hadronic collisions, J. High Energy Phys. 09 (2015) 106.

[30] For simplicity, we use the standard small- $x$ formulation where the Wilson lines are extended to $\xi=-\infty: U_{x}^{R}=[\infty,-\infty]_{x}^{R}$.

[31] Note that there is a nonscattering contribution as well in the definition of the operator in Eq. (58), which we subtracted.

[32] G. Beuf, Dipole factorization for DIS at NLO: Combining the $q \bar{q}$ and $q \bar{q} g$ contributions, Phys. Rev. D 96, 074033 (2017).

[33] H. Hänninen, T. Lappi, and R. Paatelainen, One-loop corrections to light cone wave functions: The dipole picture DIS cross section, Ann. Phys. (Amsterdam) 393, 358 (2018).

[34] Here the covariant derivative $D_{b}^{i} U_{b}$ is to be understood as an operator acting on everything on its right, not to be mistaken for $\left(D^{i} U\right)_{b}$. 